\title{
La maintenance des automotrices RER à la RATP, de Montsouris à Sucy
}

The maintenance of the self-propelled RER of the RATP, from Montsouris to Sucy

Jean-Pierre Boudrie

\section{OpenEdition}

\section{Journals}

Édition électronique

URL : https://journals.openedition.org/rhcf/1781

DOI : 10.4000/rhcf.1781

\section{Éditeur}

Rails \& histoire

\section{Édition imprimée}

Date de publication : 1 décembre 2003

Pagination : 320-325

ISBN : 0996-9403

ISSN : 0996-9403

\section{Référence électronique}

Jean-Pierre Boudrie, "La maintenance des automotrices RER à la RATP, de Montsouris à Sucy »,

Revue d'histoire des chemins de fer [En ligne], 28-29 | 2003, mis en ligne le 30 décembre 2014, consulté le 22 avril 2022. URL : http://journals.openedition.org/rhcf/1781 ; DOI : https://doi.org/10.4000/rhcf. 1781 


\section{La maintenance des automotrices RER à la RATP, de Montsouris à Sucy}

À l'origine, les automotrices de la ligne B étaient entretenues de façon très classique : entretien au temps, chaque rame revenant à l'atelier selon une périodicité prédéterminée.

La révision était traitée par voiture, chacune faisant l'objet d'une révision limitée ou d'une révision générale également selon des périodicités, c'est-à-dire que c'est le temps qui déterminait l'entretien et non l'état réel du matériel.

Aujourd'hui, les automotrices de la ligne B sont entretenues par l'Unité de maintenance du RER, dans une organisation basée sur un dépannage le plus réactif possible, situé au plus près de la ligne, et une maintenance visant à garantir une disponibilité optimale du matériel au plus juste coût.

L'Unité directrice du matériel roulant RER a en charge un parc de :

- 125 éléments de trois voitures MS61, matériel mono-courant $1500 \mathrm{~V}$ à JH conçu dans les années 1960 (fig. 1) ;

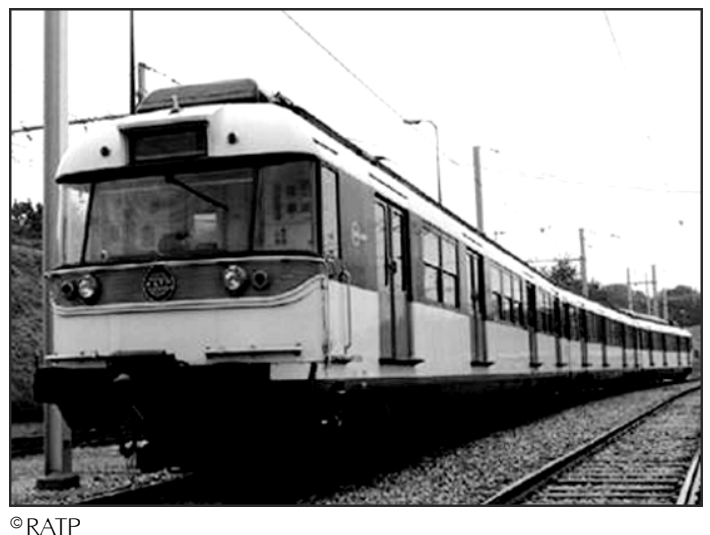

Figure 1. Le matériel MS61 en ligne (RER B). Coll. et cl. RATP. 
- 119 éléments de quatre voitures MI79, matériel bi-courant $1500 \mathrm{~V} / 25 \mathrm{kV}$ à caisse aluminium et hacheur de courant conçu dans les années 1975 dont 51 appartiennent à la SNCF (fig. 2) ;

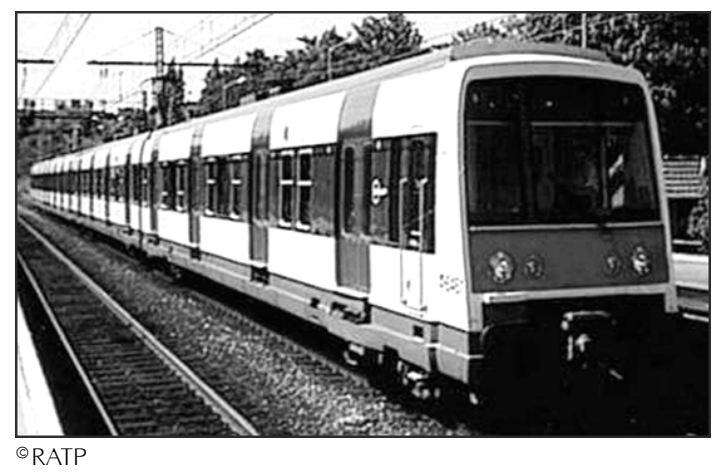

Figure 2. Une rame MI79 (RER B). Coll. et cl. RATP.

- 73 éléments de quatre voitures MI84, matériel bi-courant $1500 \mathrm{~V} / 25 \mathrm{kV}$ à caisse aluminium et hacheur de courant conçu dans les années 1980) (fig. 3);

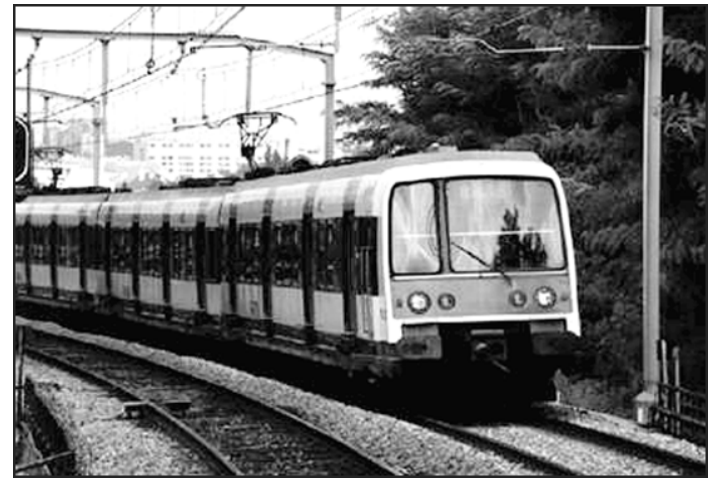

๑RATP

Figure 3. Le matériel MI84 (RER B). Coll. et cl. RATP. 
- 29 éléments de cinq voitures de MI2N, matériel bi-courant $1500 \mathrm{~V} / 25 \mathrm{kV}$ à caisse acier à deux niveaux et onduleur de courant conçu dans les années 1990 (fig. 4);

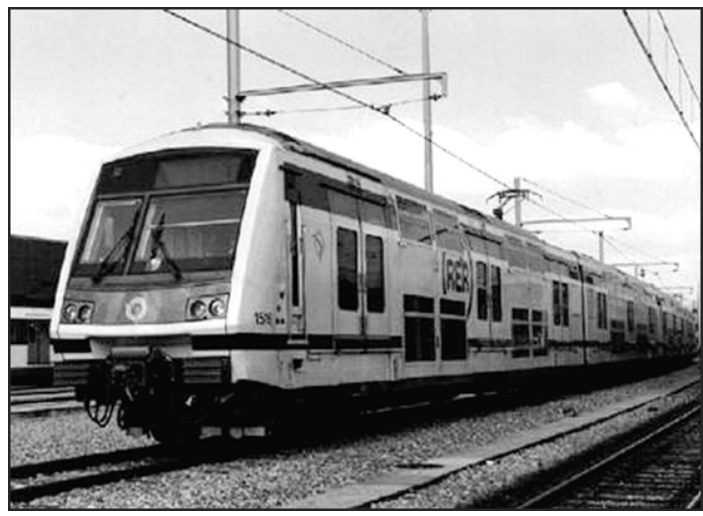

${ }^{\circ}$ RATP

Figure 4. Les voitures MI2N (RER B). Coll. et cl. RATP.

- une centaine de "VMI », véhicules de maintenance des infrastructures (locotracteurs Diesel-électriques, draisines, plates-formes...) (fig. 5).

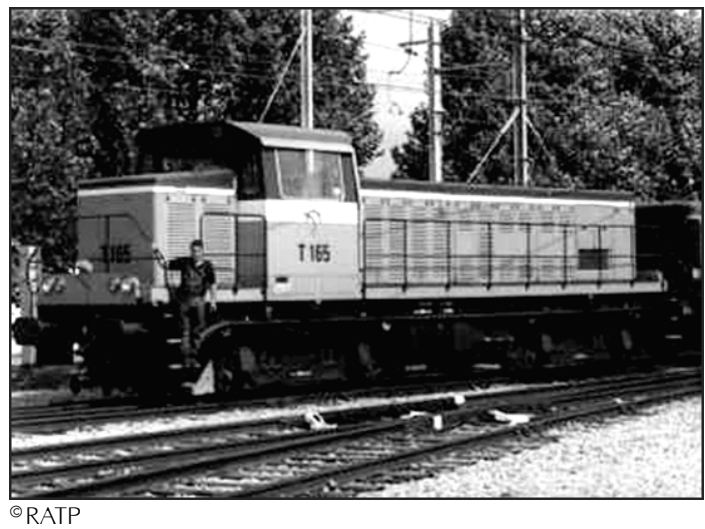

Figure 5. Locotracteur Diesel-électrique utilisé pour la maintenance des infrastuctures de la ligne B du RER. Coll. et cl. RATP. 
La maintenance des matériels voyageurs est organisée dans :

- des centres de dépannage des trains (CDT) ;

- des ateliers de maintenance des trains (AMT) assurant les dépannages lourds et la maintenance au quotidien ;

- des ateliers dits de révision patrimoniale.

Le schémas ci-après montre la répartition des installations le long des lignes A et B (fig. 6).

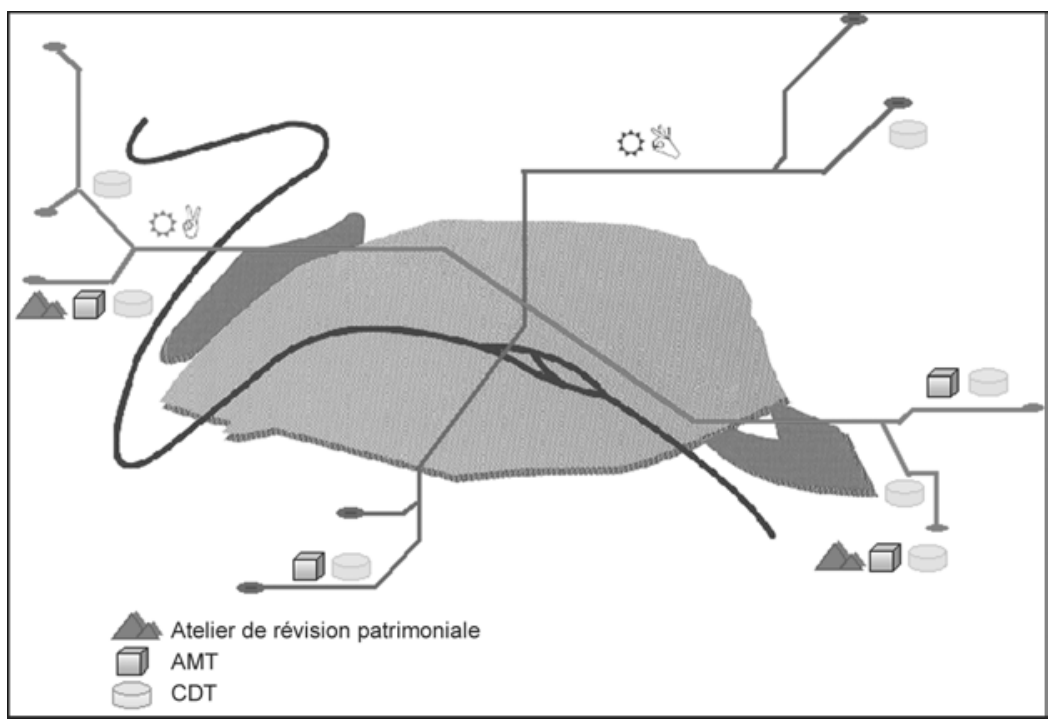

Figure 6. Les installations de dépannage, maintenance et révision du RER. ๑RATP.

Les Centres de dépannage sont répartis le long des lignes à proximité de faisceaux de garage afin de gagner en temps de réaction,

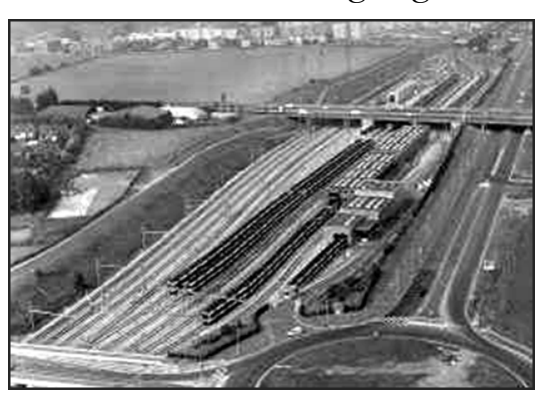
l'objectif étant une immobilisation minimale des rames en cas d'avarie. $80 \%$ des pannes y sont traitées en moins d'une heure (fig. 7).

Figure 7. Le centre de dépannage de Torcy en 1993. Coll. RATP, cl.

๑RATP-Gérard Dumax RATP/Gérard Dumax. 
La maintenance au quotidien est organisée par rame, selon des pas kilométriques, comprenant des opérations systématiques et des opérations cycliques (fig. 8).

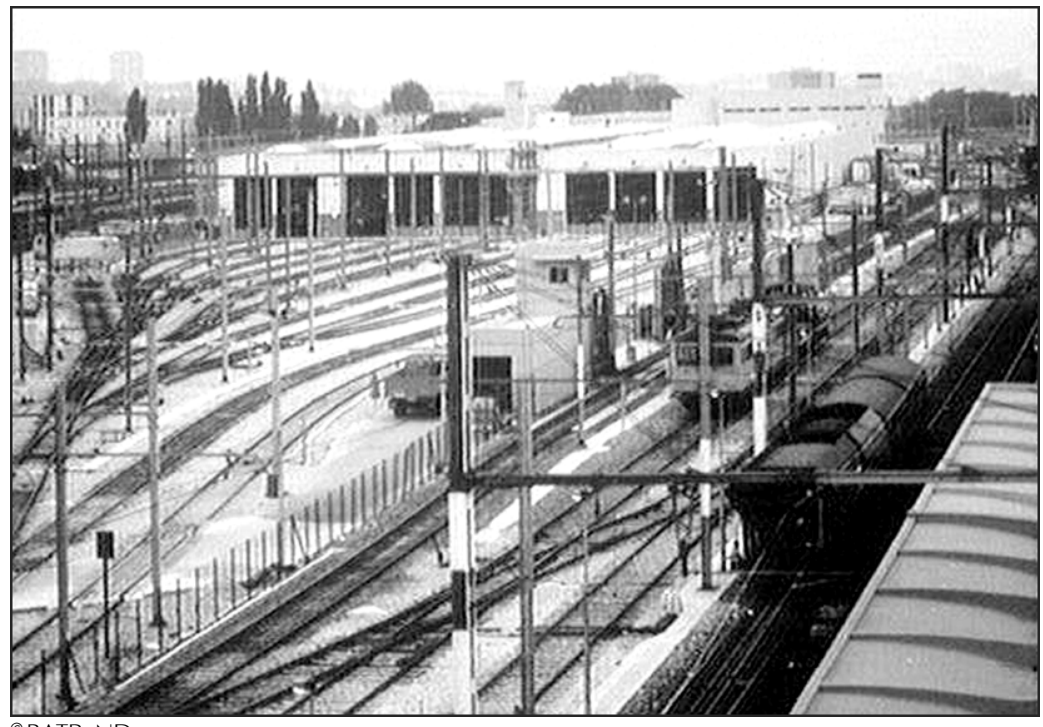

Figure 8. Le centre de Massy (atelier de maintenance des trains) en 1980. Coll. RATP, cl. RATP/ND.

La maintenance patrimoniale s'effectue « à l'organe », chaque organe du matériel ayant une périodicité propre, au kilomètre ou au temps selon sa nature (fig. 9).

Figure 9. Le centre de Sucy (atelier de révision patrimoniale) en 1993. Coll. RATP, cl. RATP/Gérard Dumax.

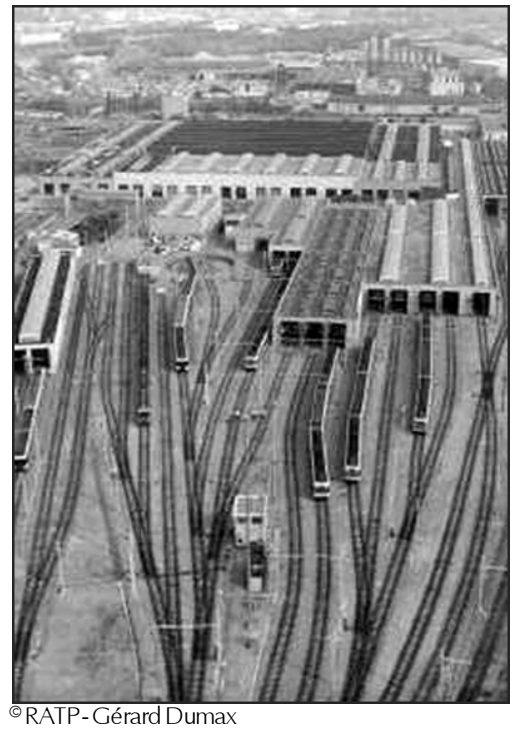


L'évolution des pas de maintenance est validée par des suivis semestriels de comportement du matériel.

La qualité joue un rôle très important dans le fonctionnement de l'unité, totalement certifiée ISO 9001 version 2000. Un élément très positif d'évolution a été la volonté de management par la qualité totale, validé par la participation au prix du Mouvement français pour la qualité (MFQ). L'unité a remporté le prix Ile-de-France en 1998, puis a été lauréate au prix national en 2001.

Cette organisation ainsi que la démarche qualité ont permis d'obtenir une amélioration constante de la qualité de service des deux lignes, ainsi que le prouvent les courbes jointes (fig. 10 et 11).

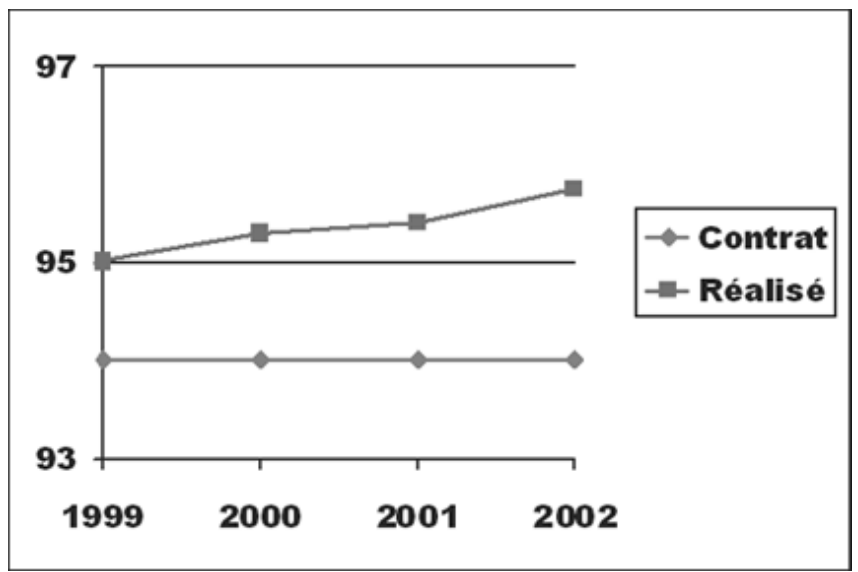

Figure 10. Qualité de service sur la ligne B du RER, 19992002. Source : RATP.

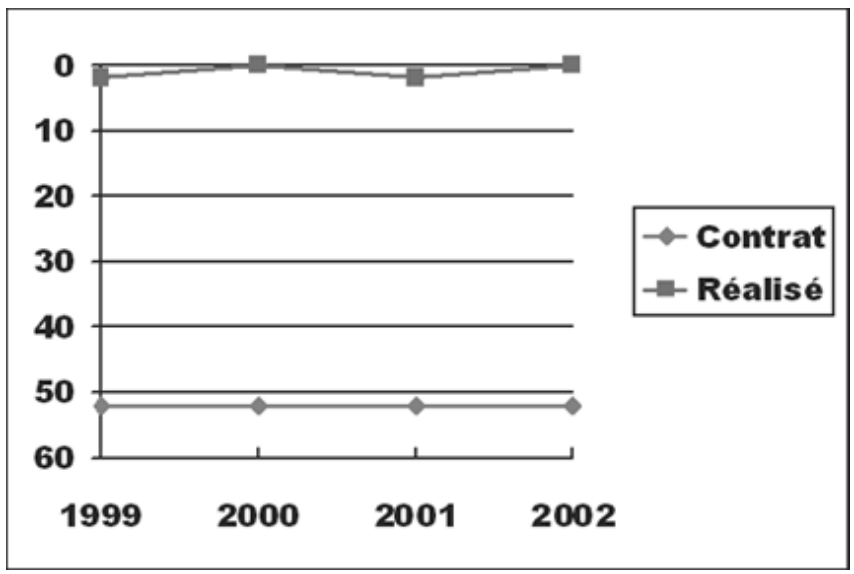

Figure 11. Missions supprimées pour manque de matériel sur la ligne B du RER, 1999-2002. Source : RATP. 